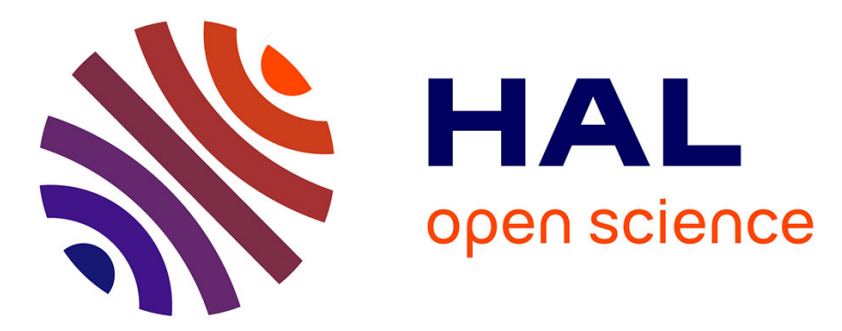

\title{
Convergecast in Wireless Body Area Networks
}

Wafa Badreddine, Nesrine Khernane, Maria Potop-Butucaru, Claude Chaudet

\section{To cite this version:}

Wafa Badreddine, Nesrine Khernane, Maria Potop-Butucaru, Claude Chaudet. Convergecast in Wireless Body Area Networks. Ad Hoc Networks, 2017, 66, pp.40-51. 10.1016/j.adhoc.2017.08.008 . hal01301773

\section{HAL Id: hal-01301773 \\ https://hal.science/hal-01301773}

Submitted on 12 Apr 2016

HAL is a multi-disciplinary open access archive for the deposit and dissemination of scientific research documents, whether they are published or not. The documents may come from teaching and research institutions in France or abroad, or from public or private research centers.
L'archive ouverte pluridisciplinaire HAL, est destinée au dépôt et à la diffusion de documents scientifiques de niveau recherche, publiés ou non, émanant des établissements d'enseignement et de recherche français ou étrangers, des laboratoires publics ou privés. 


\title{
Convergecast in Wireless Body Area Networks*
}

\author{
Wafa BADREDDINE*, Nesrine KHERNANE ${ }^{\ddagger}$, Maria POTOP-BUTUCARU* \\ and Claude CHAUDET ${ }^{\dagger}$ \\ *UPMC Sorbonne Universités,LIP6-CNRS UMR 7606, France \\ FirstName.LastName@lip6.fr \\ $\ddagger$ Franche Comte University, FEMTO-ST, CNRS UMR 6174 \\ khernanenesrine@gmail.com \\ ${ }^{\dagger}$ Institut Mines-Telecom, Telecom ParisTech, CNRS LTCI UMR 5141 \\ claude.chaudet@telecom-paristech.fr
}

\begin{abstract}
Wireless Body Area Networks (WBAN) is a recent challenging area in the domain of health monitoring. There are several concerns in this area ranging from energy efficient communication to designing delays efficient protocols that support nodes dynamicity induced by human body mobility. This paper focuses on the convergecast or data gathering protocols in WBAN. Our contribution is twofold. First, we extensively analyze the impact of postural body mobility on various classes of multihop convergecast strategies. Our study does not limit itself to the existing state-of-the-art in WBAN, we adapted to WBAN settings strategies from the areas of Delay Tolerant Networks (DTN) and Wireless Sensor Networks (WSN). We evaluate all these strategies via the OMNeT++ simulator that we enriched with realistic human body mobility models and channel models issued from the recent research on biomedical and health informatics. Our simulations show that existing results in DTN and WSN cannot be just extrapolated to WBAN without a deeper investigation. That is, existing convergecast strategies for DTN or WSN do not perform well with postural body movements because of the topological partitioning provoked by important link attenuations due to signal obstructions by clothes or body itself. Our extensive simulations give valuable insights and directions for designing efficient convergecast adaptive strategies in WBAN. Second, we propose and evaluate two novel classes of convergecast strategies.

The first class integrates in the routing information the link attenuation while the second one exploits multi (pre-established) paths to the sink node. We stress all the strategies under a realistic channel model and present an extensive analysis in terms of resiliency to mobility, end-to-end delay and energy consumption for seven different mobility patterns. We advocate that, so far, there is no strategy for convergecast in WBAN that optimizes all the above criteria for all possible mobility patterns. However, each considered strategy can be a good candidate for a specific combination of parameters with a specific mobility pattern.
\end{abstract}

\section{INTRODUCTION}

Wireless Body Area Networks (WBAN) is an already established interdisciplinary area linking mainly Wireless Sensor Networks (WSN) and Delay Tolerant Networks (DTN). A WBAN ([9], [16]) is a wireless interconnected set of sensors used to monitor, collect and transmit medical signs and other physiological parameters. WBAN have a wide area of applications such as remote health monitoring, military, security, sports and gaming. In this work we are interested in WBAN where sensors are placed on the body. In these type

\footnotetext{
*This work was funded by SMART-BAN project (Labex SMART)
}

of networks sensors performances are tremendously influenced by the body mobility and wearing clothes.

WBAN differs from typical large-scale WSN ([1], [13]) in several aspects: the size of the network is limited to generally less than twenty sensors, in-network mobility follows the body movements and the wireless channel has its specificities. The communication links in WBAN have, in general, a very short range and a quality that varies with the body posture, but remains low in order to reduce electromagnetic exposition. Consequently, the effects of body absorption, reflections and interferences cannot be neglected. Therefore it is difficult to maintain a direct link (one-hop) between a data collection sink and all WBAN nodes. Multi-hop communication represents a viable alternative and most of the recent research in WBAN focuses on such communication protocols. Short range transmission and postural movements in WBAN have a tremendous impact on the network partitioning implying a kind of body Delay Tolerant Network (DTN) ([6]). Delay-Tolerant Networks protocols seem particularly relevant in this context, as they are designed to tackle the intermittent connectivity and unpredictable signal blocking and attenuation. However, DTN usually suppose either an almost perfect knowledge of the mobility patterns or a fully random mobility. In WBAN the body postural mobility do not match the mobility models usually considered in DTN hence the performance of the protocols optimized for DTN may have poor results in WBAN.

a) Our contributions.: In this paper, we concentrate on the sole convergecast problem. We analyze the behavior, face to realistic channel models, of various convergecast strategies adapted from WSN and DTN literature and propose two novel classes of strategies from the analysis of the strengths and weaknesses of the different approaches. We compare resiliency to the body mobility, completion delay and required transmissions of more than ten different algorithms over real human body mobility traces via extensive simulations.

b) Roadmap.: The paper is organized as follow: Section II presents the convergecast problem and discuss relevant related works. In Section III we present our original contribution, that we compare through simulations in Section IV with strategies adapted from WSN and DTN areas. Section IV describes the realistic channel model we implemented and finally presents and analyzes the evaluation results in terms 
of end-to-end delay, energy consumption and resiliency to mobility.

\section{RELATED WORK}

In this section we propose an overview of the existing strategies for convergecast in several areas such as Ad-hoc, DTN, WSN and WBAN. Based on the amount of knowledge that is available, the convergecast algorithms for WSN or DTN can be divided into: dissemination (gossip-based algorithms) and forwarding (overlay-based algorithms). Gossip consists in disseminating every message to every or a subset of nodes in the neighborhood. This strategy is adopted when no knowledge on the networks is available. The overlay-based algorithms use generally tree-based overlays (constructed either on-thefly or pre-constructed) in order to push the data from the source to the sink over pre-established paths. The extreme form of gossip is flooding where every message is set to every neighbor, hence maximizing the probability that a message is successfully transferred to its destination(s).

In their paper [24], Ni et al. focus on the broadcast problem in mobile ad-hoc networks (MANET). The problem is referred to as broadcast storm. Blind flooding generates numerous transmissions causing collisions, increased contention and redundant transmissions. Ni et al. propose five schemes to solve the broadcast problem. Unfortunately, these schemes (Counterbased scheme, distance-based scheme, location-based scheme and cluster-based scheme) are not appropriate within WBAN context for many reasons related to WBAN environment (a limited number of sensors, in-network mobility follows the body movements) or related to sensors characteristics (low battery capacity, limited computational power) which reduce their functionalities. In the following, the probabilistic scheme will be adapted to WBAN context, since no extra functionality is needed. Our approach will be based only on the probability P. Results will be discussed in section IV.

Even with a probabilistic transmission, a node may receive multiple instances of the same message. For this reason, Vahdat et al., adapt another approach in [27] where they introduce the epidemic routing. They consider the non-existence of a fully connected path between the source and the destination. Based on that, the source disseminates information through basic flooding on its neighborhood and when two distant nodes eventually meet, they then exchange only the missing messages in order to forward to the destination. The exchange assumes that both nodes keep in memory the list of all received messages. In WBAN context, this approach has several limitations due to the fact that sensor nodes are characterized with limited memory capacity, adding to that the instantaneous human body mobility.

Several papers [19], [10] focus on the intermittent connection where traditional routing protocols to reach a single destination cannot be based simply on a classical shortest path algorithm. In [19], authors propose to forward the message based on a local time-space neighbors table. The results confirmed that the transmissions efficiency increases with the knowledge of the mobility pattern. In WBAN context, two constraints to deal with, basically, the proposed algorithm is heavy comparing to our sensors characteristics on term of computational power, adding to that a special mobility model based on an instantaneous human body mobility.

In [10], authors focus on DTN routing problem taking into account special constraints as: a finite buffer at each node and the fact that an end-to-end path may ever exist between a source and a destination. Depending on the amount of knowledge about the network, Jain et al. propose three classes of algorithms: zero knowledge algorithms, complete knowledge algorithms and partial knowledge algorithms. These algorithms were compared within two scenarios: routing to remote village and network of city buses. The environment and the scenarios used to evaluate these algorithms are too different from WBAN context. WBAN is characterized with a limited number of nodes ( 7 in our case). Each node is considered as a source and each source generates a single message to be forwarded to the sink.

In [11], [14] and [12], authors focus on reliability problem i.e. data loss as a major constraint in WSN and are interested in particular energy efficient routing protocols.

In their paper, Grover et al. focus the issues of SPIN (Sensor Protocol for Information via Negotiation) routing protocols which are data centric protocols. Authors discuss different versions of SPIN routing protocols. The approach to forward data from a source to a destination is similar to our approach used in attenuation-based protocols (see section III) as it is based on a three-way handshake between the source and its neighbors. We are practically interested in the modified version of SPIN: M-SPIN [25]. In M-SPIN, node transmits data to the closest node to the sink, the distance is calculated on the basis of hop distance. In our case, the metric is the attenuation of the link between the potential next hop nodes and the sink. In the context of WBAN, the distance is not an expressive metric for two main reasons: First, the limited number of nodes i.e. a limited number of hops, second, the instantaneous mobility of human body.

In addition of studying SPIN protocols and its various versions, Sharma et al present a comparative study between flooding and SPIN protocol. In our context different versions of flooding, referred to as gossip-based algorithms, were studied as flooding present former strategy to forward data into a network.

Several routing protocols have been proposed for WBAN that can be classified with respect to their aims in different categories [20], [21]: temperature based routing protocols, cross layer, cost-effective routing protocols, QoS-based routing protocols. Most of these proposals are not suitable when sensors are external (on the body and not in the body). DTN like solutions [2] and many other WBAN proposals (such as EDSR [22]) do not take into account the mobility of the human-body: during the transmissions of the message over the path already computed, disconnections can happen causing failures. In a very recent work we propose new broadcast strategies in WBAN [3].

A different research direction on the convergecast topic in 
WBAN is the convergecast on top of tree-based overlays. Several cross layer protocols have been proposed [26], [17], [29], [4]. However, these protocols have not been tested face to real pattern mobility or channel models for WBAN since at the time of their publication there were no available models.

Multipath routing in WSN has been considered as an important field of research and widely used to improve network performance considering reliability, security and load balancing. The authors of [18] propose an efficient N-To-1 multipath discovery protocol to find multiple paths from every sensor node to the sink in two route discovery phases, i.e. route update and multipath extension flooding phases. In the route update flooding phase, a spanning tree is constructed by broadcasting the route update message. At the reception of this message the node set the sender node as parent node. Then in the multipath extension phase an alternative path is created by the exchange of messages between the nodes that belong to different branches of the spanning tree. However in the data transmission stage the source node splits its data into multiple packets to be sent over the different paths, which can results in loss of the whole data if a significant packet is lost. In addition the N-to-1 multipath routing protocol uses the flooding strategy during the route discovery phase, thus leading to a significant consumption of nodes' energy, and additional overhead in the network especially in the context of WBAN networks. To cope with energy and collision problems, the authors of [28] propose a multipath routing algorithm that uses two collision-free paths between the source and sink nodes.

Although their mechanisms help to conserve much more energy compared to [18], it cannot be used in a WBAN context for two reasons: the postural mobility and impossibility to construct two collision free paths.

Recently, the apparition of realistic channel models and more generally the cross domain research between electronics and computer science creates the premises to realize realistic simulations for WBAN and stressing the existing protocols under realistic settings. Our work focuses on flat (i.e. non cross-layer) convergecast strategies. We stress strategies inspired from the WSN and DTN literature under realistic model channel for WBAN [23]. Then, we propose two novel classes of strategies that improve over existing one in terms of endto-end delay and resilience to the body mobility.

\section{Convergecast Strategies}

This section proposes a detailed description of different convergecast strategies we compare in this paper. We describe below gossip-based strategies and two novel classes of convergecast strategies.

The first class depends on a minimum knowledge of the network to take the decision either to forward the message or not and to which node. According to the mobility model described below IV-A, we will use the path loss value (the attenuation term is used in the following sections) of the link between each node and the sink (similar to the way the classical geographical routing uses the distance to the sink).
The second class exploits predetermined paths between the source and the sink.

We have chosen only gossip-based strategies for comparison since popular strategies such as [7] and [5] have poor performances in all the mobility patterns we considered ${ }^{1}$.

1) Gossip-based strategies: In this category, no information about the network is used. Messages are forwarded to the sink based on a broadcast. The strategies are inspired from the state of the art in WSN and DTN and our recent work related to broadcast in wireless body area networks [3]. Three main strategies are evaluated:

- FloodToSink: The source $S$ broadcasts the message. Each node, except the sink, that receives the message, rebroadcasts it while the message TTL (Time to Live) is greater than one.

- ProbaCvg: The source $S$ broadcasts the message. When receiving the message, each node, except the Sink, decides whether it should broadcast the message or not depending on a probability $P>0$. For all nodes, $P$ is set initially to 1.0 . For broadcasting, a node chooses a random variable $r$ and compares it to the value of $P$, if it's lower than $P$ than the node decides to broadcast, otherwise it discards the message. After each broadcast, the node divides $P$ by 2 .

- PrunedCvg: The source $S$ broadcasts the message. When receiving the message, each node, except the Sink, chooses randomly the node to which it forwards the message.

2) Attenuation-based strategies: In this section we describe four different convergecast strategies. All of them exploit information about the link attenuation (path loss) provided by the channel model we used and described in section IV-A. Each node has an estimation of its path loss value to all nodes. The common pattern followed by the four strategies when a source $S$ wants to send data to the sink is described on Figure 1 .

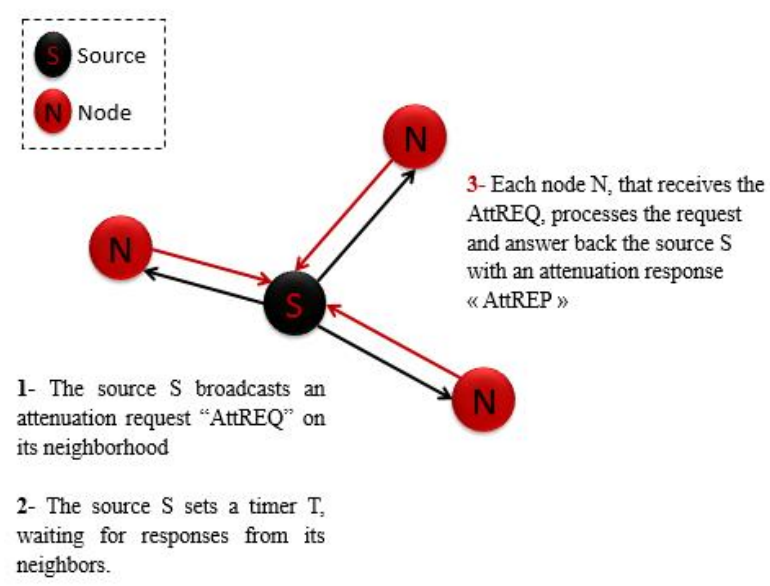

Fig. 1: Attenuation assessment procedure

\footnotetext{
${ }^{1}$ Due to lack of space the comparison with these strategies was not included in the paper.
} 
The source starts by broadcasting a request "AttREQ" asking its neighbors about the attenuation value of their connection to the sink and activates a timer $T$. After timer $T$ expiration, the source $S$ checks if any received responses:

- If no response is received, the source rebroadcasts an attenuation request "AttREQ".

- If $S$ gets a response from one or more neighbors, the source processes the responses, thus we have four strategies:

1) MinAtt: The source selects the node that corresponds to the smallest link attenuation to the sink. In other words, source transmits the message to the node of which link with the sink is the most reliable.

2) BothMinAtt: The source chooses to send the message to two nodes. They correspond to the closest nodes to the sink based on attenuations values.

3) CloseToMe: For this strategy, in addition of asking link attenuation between each node and the sink, the source also requested the attenuation value of the link that exists between it and each node receiving the request. The source $S$ chooses the closest node to itself.

4) RandAtt: The source $S$ chooses the next hop based on a random choice between all nodes that answered its request.

- Finally, the node designated to forward the source data message, will follow the same process to designate the next hop.

3) Multi-path strategies: In this section, a second novel class of strategies is presented. We uses information about the network to define these strategies. We referred to nodes position compared to the sink and we support our choices by the mobility model we used that allows us to give an overall view of the state of links between the nodes.

Contrary to the first class, here the choice is fixed all long the simulation.

In this class, each node has two pre-selected privileged neighbors (call in the sequel parents) as shown in Figure 2.

The choice of the first path is based on the shortest path to the sink in terms of number of hops (e.g. [8]), while the second path is chosen in such a way that a node will always have at least one path to the sink even in case of mobility. if we take the thigh, wrist, and navel nodes as an example, even if the communication between the thigh and wrist nodes is interrupted in case of mobility (wear posture), the distance between the thigh and navel nodes remains the same (it may even decrease if the person leans).

On top of the overlay defined above we execute four different convergecast strategies that aim to minimize the energy consumption but also to balance the load between the nodes used as relay to the sink. Note that the probability used in our strategies influences only the choice of neighbor not the decision of sending or not the data. Thus, the strategies are as follows:

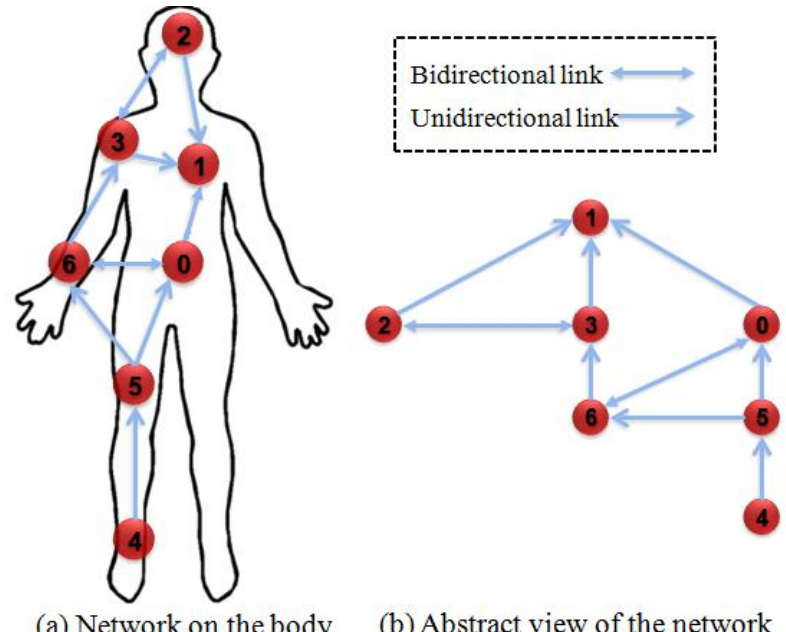

Fig. 2: Architecture of the network

1) APAP (All Parents to All Parents): The source node sends its data to all its parent nodes in the neighbors table. At the reception of the data, parent nodes verify if the sink is in their neighbors table. If yes, they send the data to the sink, otherwise, they send data to all their parent nodes.

2) APPP (All Parents to Probabilistic Parent): The source node sends its data to all its parent nodes in the neighbors table. At the reception of the data, parent nodes verify if the sink is in their neighbors table. If yes, they send the data directly to the sink, otherwise they select one of their parent nodes with a probability $P$, set to 0.5 in our simulations, i.e each parent has an even chance of being selected.

3) PPAP (Probabilistic Parent to All Parents): The source node sends its data to one of its parent nodes chosen randomly with probability $P>0$ (set to 0.5 in our simulations). At the reception of the data, the parent nodes verify the existence of the sink in their neighbors table. If it exists, they send the data directly to the sink, otherwise to all their parents defined in the table.

4) PPPP (Probabilistic Parent to Probabilistic Parent): The source node sends its data to one of its parent nodes chosen randomly with probability $P>0$ (also set to 0.5 in our simulations). At the reception of the data, the parent nodes verify the existence of the sink in their neighbors table. If yes they send the data directly to the sink, otherwise they select one of their parent nodes in the neighbors table with probability $P$.

\section{Simulation SETtings AND Results}

In order to evaluate the algorithms described in the previous section III in a realistic WBAN scenario, we implemented them under the OMNeT++ simulator. OMNeT++ includes a set of modules that specifically model the lower network layers WBAN through the Mixim project [15]. We stress the above 
strategies under a realistic channel model [23] with several types of postural movements.

\section{A. Channel model}

We decided to implement a realistic channel model published in [23] over the physical layer implementation provided by the Mixim framework. This channel model of an on-body $2.45 \mathrm{GHz}$ channel between 7 nodes, that belong to the same WBAN, using small directional antennas modeled as if they were $1.5 \mathrm{~cm}$ away from the body. Nodes are assumed to be attached to the human body on the head, chest, upper arm, wrist, navel, thigh, and ankle.

The nodes positions are calculated in 7 postures: walking (walk), walking weakly (weak), running (run), sitting down (sit), wearing a jacket (wear), sleeping (sleep), and lying down (lie). Walk, weak, and run are variations of walking motions. Sit and lie are variations of up-and-down movement. Wear and sleep are relatively irregular postures and movements.

Channel attenuation is calculated between each couple of nodes for each of these positions as the average attenuation (in $\mathrm{dB}$ ) and the standard deviation (in $\mathrm{dBm}$ ). The model takes into account: the shadowing, reflection, diffraction, and scattering by body parts, but interference from other WBAN systems.

Naganawa et al. studied in their paper [23] a cooperative transmission scheme: two-hop relaying scheme. Using the simulated path loss, the performance of such scheme were evaluated by comparing the outage probability using different relay nodes against a direct link between a source and a destination. Results show that a better choice is to use a multihop communication instead of onehop, adding to this, a significant decrease of the transmission power. These results also justify the design of our multipath based strategies.

\section{B. Simulation Settings}

Above the channel model described in the previous section, we used standard protocol implementations provided by the Mixim framework. In particular, we used, for the medium access control layer, the IEEE 802.15.4 implementation. The sensitivity levels, header length of the packets and other basic information and parameters are taken from the 802.15.4 standards.

Each data point is the average of 50 simulations run with different seeds. The simulations are performed with only one packet transmission from each node except the gateway or sink node. The transmission power is set at the minimum limit level $-60 \mathrm{dBm}$ that allows an intermittent communication given the channel attenuation and the receiver sensitivity $-100 \mathrm{dBm}$. With this value of transmission power, we guarantee that at each time $t$ of the simulation, we have a connected network and at the same time we ensure a limited energy consumption.

\section{Simulation results}

To compare between the different convergecast strategies we defined in the previous section III, we studied for each one three basic parameters:
- Percentage of received messages: Throughout the simulation, each node sends one message to the sink, we therefore calculate the number of received messages by the sink being presented as a percentage in the graphs below.

- End-to-end delay: The average End-to-end delay represents the average time a message takes to reach the final destination (i.e. the node $\operatorname{sink}$ ).

- Number of transmissions and receptions:This is a key parameter, it helps us to have an estimation of the energy consumption at each node and for each posture for all the strategies.

1) Percentage of received messages: We will start by comparing the percentage of received messages for each strategy in function of body posture, presented in Figure 4. Figure shows that strategies behave differently depending on the wearer posture.

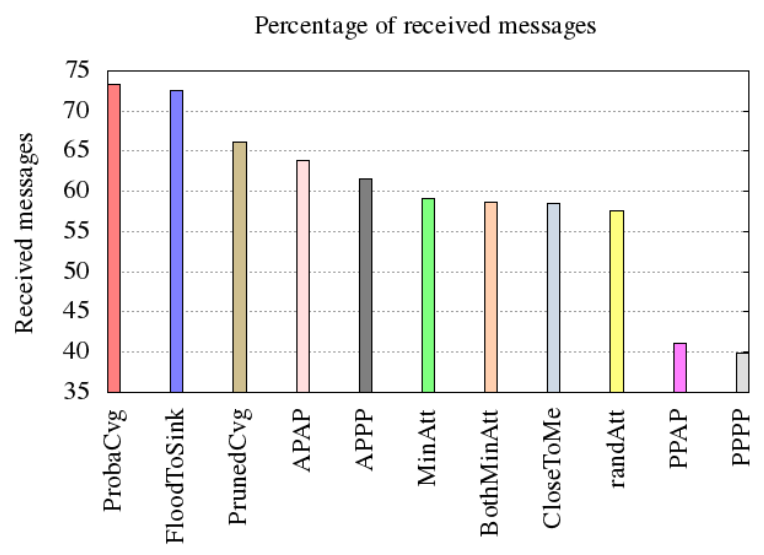

Fig. 3: Average percentage of received messages

All strategies present low performances in Sleep posture. In addition of being a static posture where there is less connections and disconnections between nodes, with this position, some nodes are hidden by different body parts and remain so all along the simulation.

FloodToSink strategy is the less affected by human body posture. The percentage of received messages varies between 60 and $81 \%$. For many postures, it has the maximal percentage, especially for "SLEEP" posture where a notable difference with the other strategies is remarkable. However, its performance decreases in RUN posture, the large amount of packets in the network causes collisions and packets loss. In this strategy, there is no condition to stop rebroadcasting the message except its TTL (Time To Live) parameter, which is set to 6 in our simulations.

For ProbaCvg strategy, a gossip-based strategy, rebroadcasting the message depends on the comparaison between the variable $r$ (chosen randomly before rebroadcasting any message) and the probability $P$ (a local variable divided by 2 after each rebroadcast). Thus, rebroadcasting the message is limited throughout the simulation. As a consequence, the 

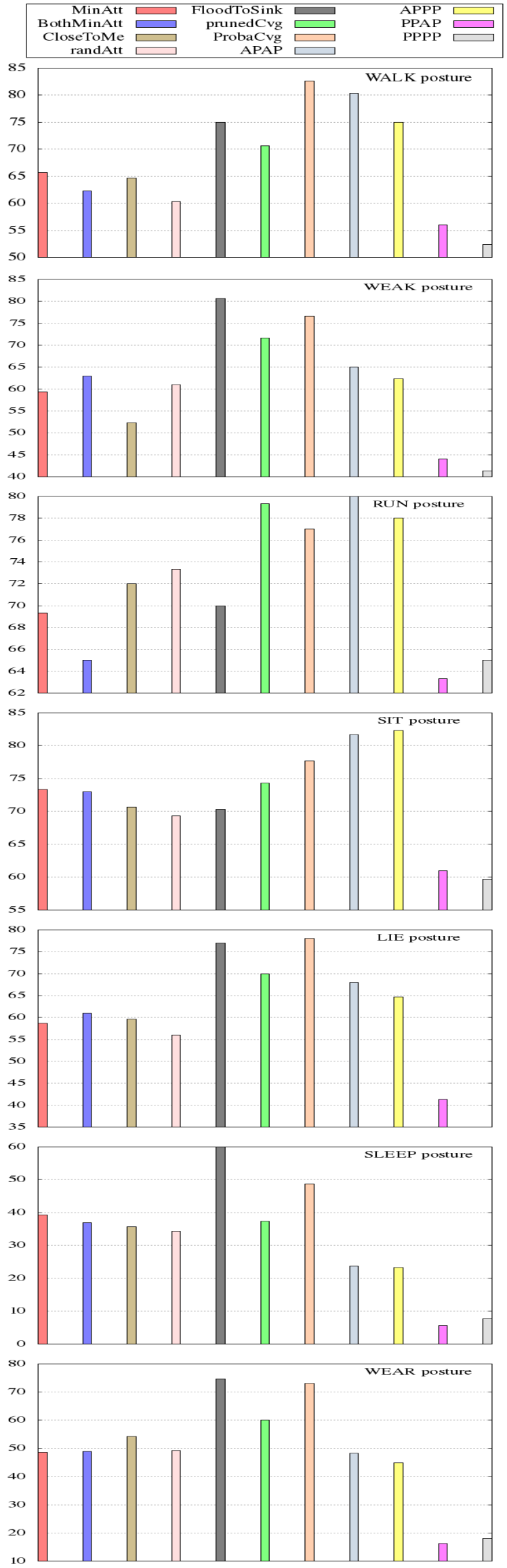

Fig. 4: Percentage of received messages number of message copies in the network decreases as well as collisions for some postures, which is reflected in a higher percentage of received message comparing to FloodToSink strategy.

Strategy PrunedCvg shows a good percentage of received messages. Remember that in this strategy, node forwards the message to a randomly chosen node. Compared to FloodToSink strategy, PrunedCvg strategy presents higher percentage for Run and Sit postures. Indeed, forwarding the message is uniformly balanced between different nodes which reduces the number of message copies in the network and thus prevents collisions and packets loss.

Contrary to the expectation, attenuation-based strategies present, in almost all postures, the lowest percentage of received messages. Even if nodes try to forward message based on a minimum knowledge of the network, this does'nt provide a high reception rate. This is due to collisions between control packets (requests and responses) and data packets.

Let us focus two strategies: a gossip-based strategy "PrunedCvg" and an attenuation-based "RandAtt", both of them are based on a random choice of the next hop. With PrunedCvg, the decision is made locally for direct sending of data message. For RandAtt, a phase of control messages exchange is needed for decision making. Results show a low percentage of received message as consequence of control and data packets collision.

Note that attenuation-based strategies results are close to each other. This is not the case for multi-path based strategies. Indeed, $P P A P$ and $P P P P$ strategies present the lowest percentage between all the considered strategies for all postures. However, APAP and APPP present good percentage of received messages. It competes with that presented by gossip-based strategies and exceeds the percentage presented by attenuation-based strategies for all postures.

2) Completion Delay: Figure 5 presents the average endto-end delay, the time a message takes to reach the sink. The delay for all strategies is affected by postural mobility. In static postures, as "Sleep", messages spend more time to reach the sink than in postures with higher mobility, as "Run".

Gossip-based strategies show better delay than attenuationbased strategies. Indeed, in attenuation-based strategies, a node cannot forward the message unless it received responses from its neighbors, otherwise, it rebroadcasts its request and delays forwarding data message until receiving at least one response.

Attenuation-based strategies are more affected by body posture than gossip-based strategies. For example, consider minAtt strategy from attenuation-based class and ProbaCvg from gossip-based class. For "Sit" posture, end-to-end delay for both strategies is respectively equal to 0.31 and 0.23 , values are close. However, for "Wear" posture, the end-to-end delay increases for minAtt strategy to 0.54, and for ProbaCvg to 0.27 . In this case the gap between the two strategies delay is more relevant.

For multi-path strategies, we observe different behaviors for the four strategies. APAP and APPP strategies have the lowest end-to-end delay. In these two strategies, source node 

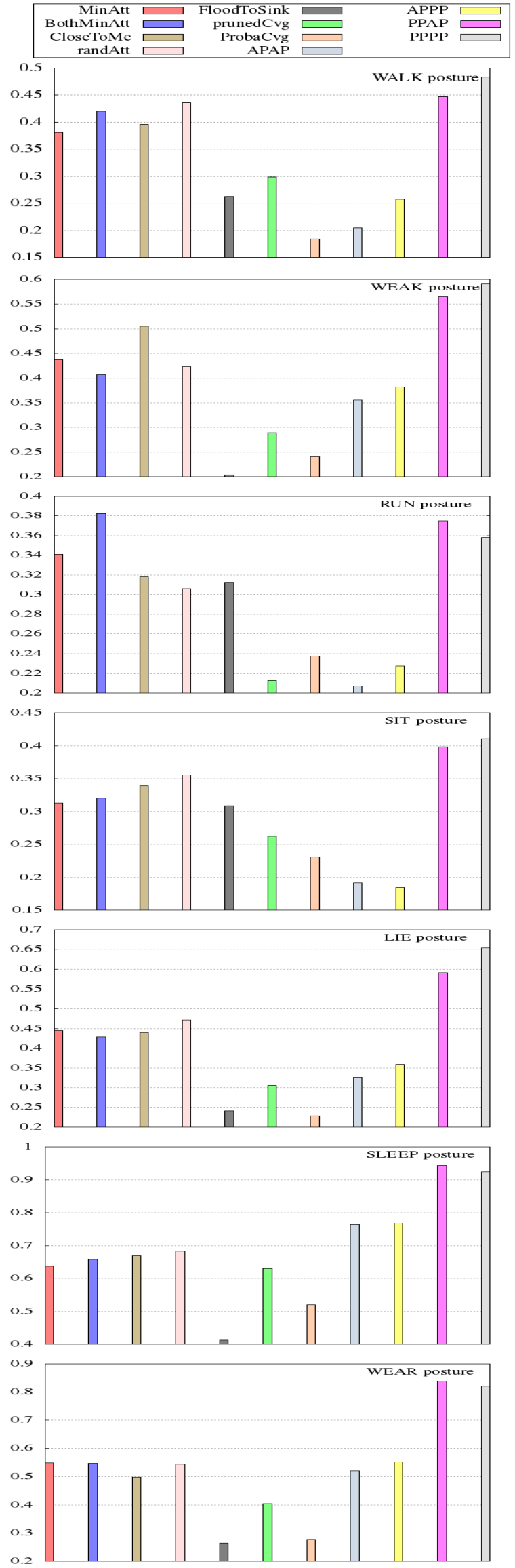

Fig. 5: Average End-To-End Delay exploits all the existing paths in its neighbors table to reach the sink contrary to a probabilistic choice in $P P A P$ and $P P P P$ strategies. A randomly chosen parent can be farther from the sink compared to the initiating node which increases the delay to reach the sink and also the probability of packet loss.

In RUN posture, gossip-based strategies show high endto-end delay especially FloodToSink strategy. RUN posture presents the highest mobility posture: even with an intermittent connectivity, nodes are able to receive and forward messages to different neighbors which, in case of gossip-based, overloads the network and causes packets loss. Differently, in case of multipath strategies ( $A P A P$ and $A P P P$ ), forwarding packets is handled only by parents nodes.

In SLEEP posture, the observation is quite opposite. Multipath strategies present the highest end-to-end delay, even higher than attenuation-based strategies. In this static posture, parents nodes could be unreachable for sources, being compelled to forward only to parents, packets are loss and delay increases. Here, gossip-based strategies show better delay especially for FloodToSink strategy.

3) Number of transmissions and receptions: The number of transmissions and receptions is a key parameter for two reasons: First, this number reflects the channel load. Second, it gives an indication on the energy consumption of each node and hence on the devices autonomy and sensors capability to rely on a reduced size battery.

In the following, we will compare, in figure 6, the number of transmissions and receptions per posture for each strategy.

Consider first gossip-based strategies. FloodToSink and ProbaCvg strategies pay for their low end-to-end delay with a huge number of transmissions and receptions. For FloodToSink, is three times more, in average, than the other strategies because nodes continue to broadcast each received message while its TTL is greater than 1 . This number decreases with ProbaCvg strategy, due to the probability of broadcast $P$, but still high comparing to the other strategies. For RUN and SIT postures, the two strategies show the highest number despite a low percentage of received message (see Figure 4) because of lot of collisions and unnecessary retransmissions.

For PrunedCvg strategy, forwarding the packet to only one randomly chosen next hop, decreases significantly the number of transmissions and receptions. Also, it is less affected by body postures, indeed, the number varies less from a posture to another.

Attenuation-based strategies show a similar behavior considering variation in function of postures. But, we notice that BothMinAtt strategy shows the highest number. This increase is because each node forwards the message to two neighbors contrary to the other strategies. We can conclude that this strategy doesn't perform well in WBAN context since the losses in terms of number of transmissions and receptions (i.e. energy consumption) are more significant than the small gain observed in terms of end-to-end delay and percentage of received messages.

Considering multi-path strategies, the number of transmissions and receptions is reasonable. Forwarding only to parents, 

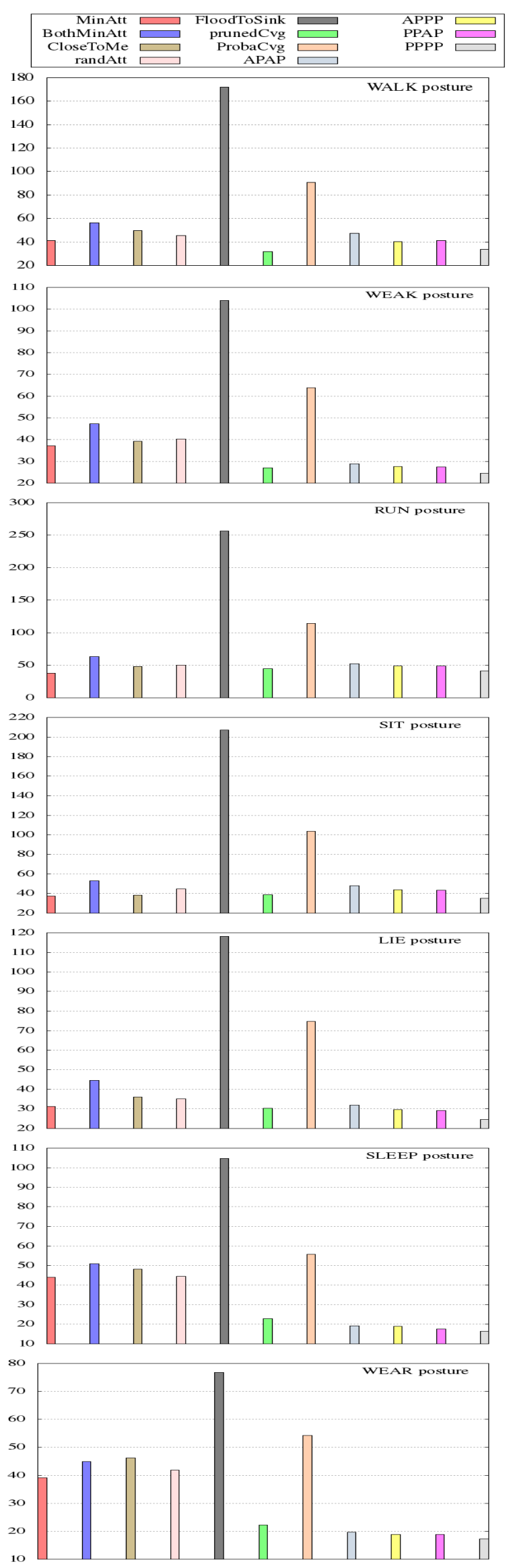

Fig. 6: Number of Transmissions and Receptions allows to control packets generation on the network.

$A P A P$ strategy has the biggest number of transmissions and receptions within all the postures comparing to the other multipath strategies because of the choice of two paths to the sink. In this strategy all existing path in a node's neighbors table are used thus more nodes are designated to forward the packet. For PPPP and PPAP strategies, a low number of transmissions and receptions doesn't mean good performance. The opposite, it confirms results about the low percentage of received messages. Few nodes are designated to forward the message.

We focus in the following two postures: "Run" and "Sleep", which represent critical postures since they have, respectively, the highest and lowest mobility.

a) Run Posture: Figure 7 shows number of transmissions and receptions per node for "Run" posture. Recall that gossipbased strategies consume more energy than attenuation-based strategies in the case of running posture (see Figure 6). In Figure 7 we show that the previous observation is not due to a particular behavior of a node. That is, nodes behave the same regardless their position on human body.

The important number of transmissions and receptions at the node in chest position is equal to only the number of receptions because in all cases, this node doesn't have to forward any message since it is the final destination.

The node at ankle position consumes less energy, for two reasons: first because it has in average one neighbor and second, for the case of attenuation-based strategies, nodes do not select this node as their best choice since it is not the closest to the sink (situated at the chest level in our simulations).

Interestingly, in "Run" posture, wrist node consumes the maximal energy. Even though this node is a peripheral node, the high mobility allows it to have different nodes in its range and hence actively participate to the convergecast.

In multipath strategies, the number of transmission and reception for ankle node refers to only the number of transmission, since it is the farthest node from the sink node in term of number of hops. So it transmits only its own data (i.e. do not have any child). The thigh and head nodes have approximately the same number of transmission and reception since they transmit their data and only the data of their child, while the navel, upper arm and wrist nodes transmits not only their own data and those of their child, but also the data of their grandchild (for example: in the APAP strategy the wrist node have to receive and transmit, in addition to its own data, the data of the ankle, thigh and navel nodes). The figure shows also that the wrist node has the highest number of transmission and reception. Although, it was expected to have the same (or approximate) number of transmission and reception with the navel node, and less than the upper arm node, the mobility of the human body in run posture is the only explanation for these results.

b) Sleep Posture: Figure 8 focuses the number of transmissions and receptions per node for "Sleep" posture.

The node at navel position has the lowest number of 

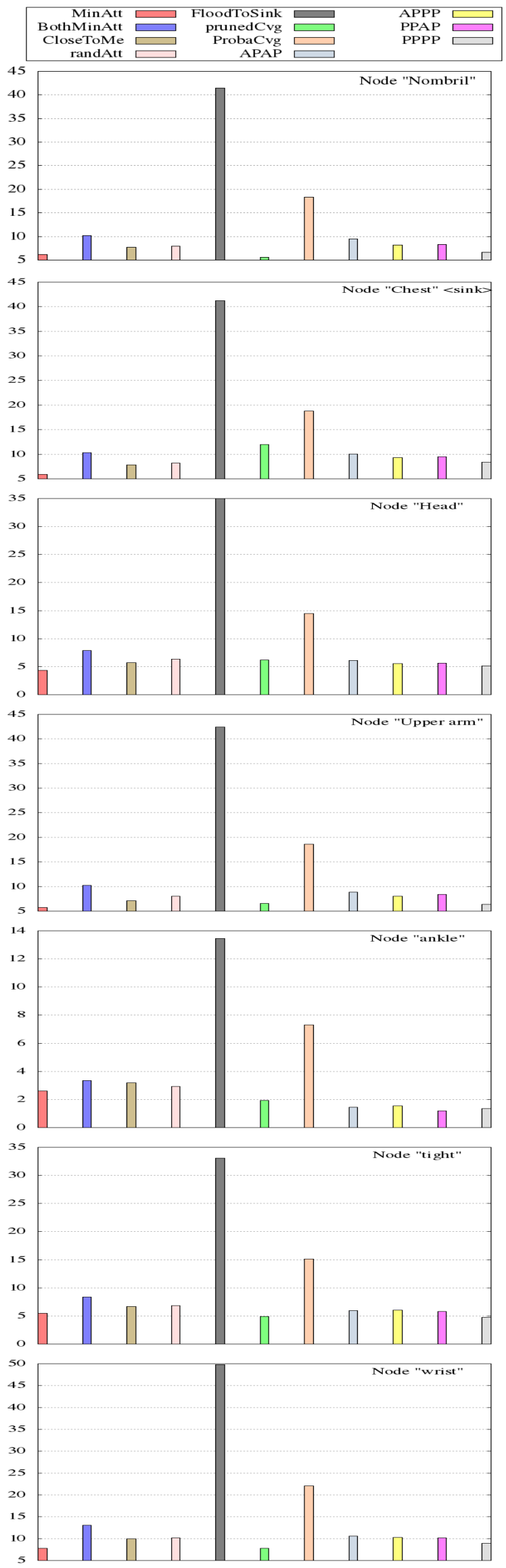

Fig. 7: Number of Transmissions and Receptions per node for "RUN" posture transmissions and receptions. Referring to our mobility model, this node is hidden during simulation.

For chest node, like for the navel node, the hidden nodes explain its low number of transmissions and receptions, in addition of not forwarding messages.

Although, the low percentage of received messages for "Sleep" posture, and the low number of transmissions and receptions at the navel and chest nodes, the number of transmissions and receptions for the rest of nodes is significant especially, for attenuation-based strategies. In this posture, even with the presence of hidden nodes, at least two nodes can see each other. Therefore, to forward a data message to the sink, a node is the only choice of its neighbor and vice versa (i.e. its neighbor is its only choice). Therefore the data message continues to oscillate between these two nodes, which increases the number of transmissions and receptions.

For multipath strategies, as mentioned previously, the navel node has the lowest number of transmission and reception due to the position of the human body at sleep posture, thus holding the node hidden for a while. Since this node has a strategic location, it was chosen as a relay node for several source nodes i.e. transmits and receives the ankle (by the intermediate of thigh node), thigh and wrist nodes data. For this reason we can clearly observe the important decreasing of the number of transmission and reception at the wrist and upper arm nodes (parents). We notice that thanks to this approach, a consumption balancing is realized between the different nodes. The behavior of nodes in this posture explains the lower performance of the multipath strategies in sleep posture on the previous graphs.

\section{Discussions}

Gossip-based strategies present in average good results. They present the highest percentage of received messages and the lowest end-to-end delay for 4 postures: WEAK, LIE, SLEEP and WEAR which corresponds to static and irregular positions. However their performances decrease in RUN and SIT postures; these two positions represent respectively the highest mobility posture and a dense network where nodes are close to each other.

In terms of number of transmissions and receptions, FloodToSink and ProbaCvg are not adequate for handling convergecast in WBAN. It is not the case for PrunedCvg strategy, a random choice of the next hop performs well in such environment. We notice a balance between percentage of received message, delay and number of transmissions and receptions.

In attenuation-based strategies nodes energy consumption is less affected by body postures than gossip-based or multipathbased strategies. However, in almost all postures, these strategies show a higher number of transmissions and receptions than multipath-based strategies. This high consumption is not compensated with a reliable convergecast or a low end-to-end delay.

To conclude, in general, for these two classes of strategies (gossip-based and attenuation-based strategies), overloading 

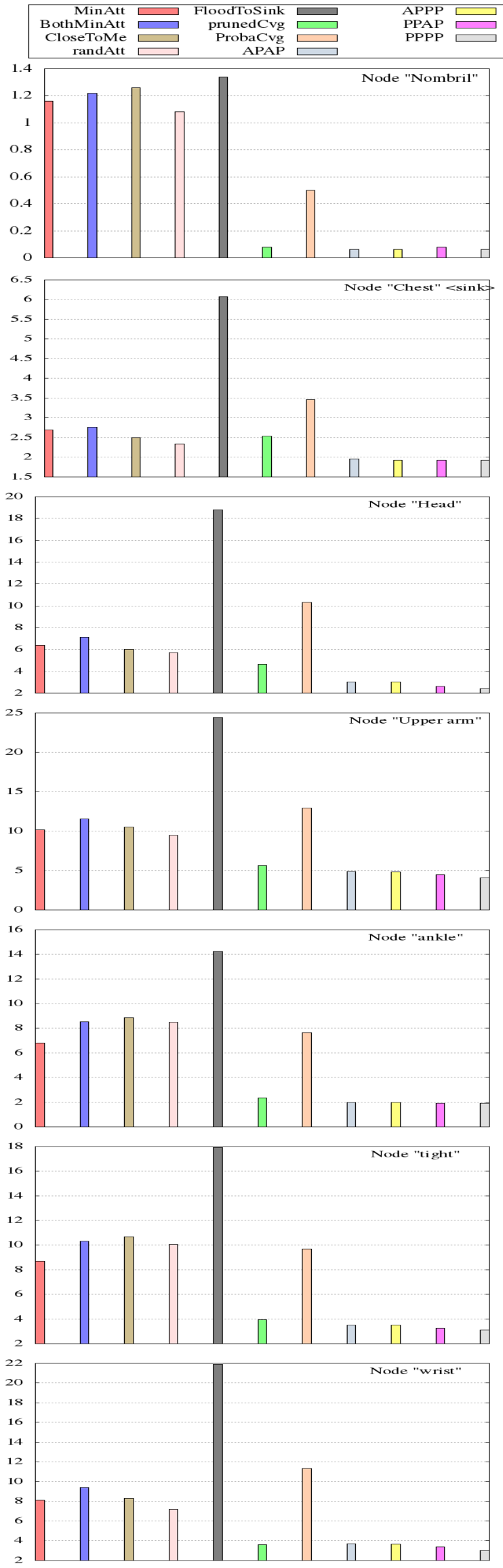

Fig. 8: Number of Transmissions and Receptions per node for "SLEEP" posture the network with packets (control or data packets), does not increase performances. On the contrary, it increases collisions, packets loss, energy consumption because of unnecessary retransmissions and network overload.

The last class of strategies "multipath-based" shows good performances. For the percentage of received messages, APAP and $A P P P$ strategies present the best percentage in Walk, Run and Sit postures. APAP shows also a good End-To-End delay (i.e. the best compared to all other strategies). It follows that this strategy is adequate for high mobility postures (Run) and dense networks (Sit). Another advantage of multipath based strategies, is the number of transmissions and receptions. Even if we exploit all existing paths with $A P A P$ and $A P P P$, the number is still reasonable compared to gossip-based strategies or attenuation-based strategies.

We can conclude that "PrunedCvg" (gossip-based strategy) and APAP (multipath-based strategy) present good results, considering the three parameters studied together. However, PrunedCvg strategy is more efficient for static and irregular postures while APAP is more efficient for high mobility posture and highly connected networks.

\section{CONCLUSIONS AND Future WORK}

In this paper we evaluated through simulation the performance of several WSN and DTN-inspired convergecast strategies altogether with two novel classes of strategies tailored for a WBAN context.

Our simulations were conducted with the OMNeT++ simulator, the Mixim framework and a WBAN realistic channel model recently proposed in the literature ([23]). The simulations realized over a 7 nodes network in 7 types of movements confirmed that some of the novel strategies achieve better performances in terms of end-to-end delay, resilience to mobility and energy consumption compared to gossip-based strategies inspired from WSN or DTN. Note that popular strategies such as [7] and [5] perform even worst in all the mobility patterns we considered ${ }^{2}$.

Several future directions are opened by our research. The first direction is to further improve the best of our strategies by combining them with old and new cross-layer techniques developed for WBAN (e.g. [26], [17], [29], [4]). Another direction is to add to the studied strategies reliability and totalorder features. Although this problem is crucial for medical applications it has never been investigated in the context of WBAN.

\section{REFERENCES}

[1] G. Anastasi, M. Conti, M. Di Francesco, and A. Passarella. Energy conservation in wireless sensor networks: A survey. Ad hoc networks, 7(3):537-568, 2009.

[2] N. T. Antonio Manuel Ortiz, Nedal Ababneh and J. Morrison. Adaptive routing for multihop IEEE 802.15.6 wireless body area networks. In 20th International Conference on Software, Telecommunications and Computer Networks, SoftCOM 2012, Split, Croatia, September 1113,2012, pages 1-5, 2012.

${ }^{2}$ Due to lack of space the comparison with these strategies was not included in the paper. 
[3] W. Badreddine, C. Chaudet, F. Petruzzi, and M. Potop-Butucaru. Broadcast strategies in wireless body area networks. In Proceedings of the 18th ACM International Conference on Modeling, Analysis and Simulation of Wireless and Mobile Systems, MSWiM 2015, Cancun, Mexico, November 2-6, 2015, pages 83-90, 2015.

[4] B. Braem, B. Latré, I. Moerman, C. Blondia, and P. Demeester. The wireless autonomous spanning tree protocol for multihop wireless body area networks. In 3rd Annual International ICST Conference on Mobile and Ubiquitous Systems: Computing, Networking and Services, MOBIQUITOUS 2006, San Jose, California, USA, July 17-21, 2006, pages $1-8,2006$.

[5] U. Colesanti and S. Santini. The collection tree protocol for the castalia wireless sensor networks simulator. Technical Report ETH Zurich.

[6] K. Fall and S. Farrell. Dtn: an architectural retrospective. Selected Areas in Communications, IEEE Journal on, 26(5):828-836, 2008.

[7] E. Ghadimi, O. Landsiedel, P. Soldati, S. Duquennoy, and M. Johansson. Opportunistic routing in low duty-cycle wireless sensor networks. ACM Trans. Sen. Netw., 10(4):67:1-67:39, June 2014.

[8] R. GhasemAghaei, A. Rahman, M. A. Rahman, and W. Gueaieb. Ant colony-based many-to-one sensory data routing in wireless sensor networks. In Computer Systems and Applications, 2008. AICCSA 2008. IEEE/ACS International Conference on, pages 1005-1010, 2008.

[9] T. Hayajneh, G. A. Al-Mashaqbeh, S. Ullah, and A. V. Vasilakos. A survey of wireless technologies coexistence in WBAN: analysis and open research issues. Wireless Networks, 20(8):2165-2199, 2014

[10] S. Jain, K. Fall, and R. Patra. Routing in a delay tolerant network. In 2004 conference on Applications, technologies, architectures, and protocols for computer communications (Sigcomm ’04), Oct. 2004.

[11] M. S. Jitender Grover and Shikha. Reliable spin in wireless sensor network: A review. IOSR Journal of Computer Engineering (IOSRJCE), 16:79-83, Nov-Dec 2014.

[12] N. M. Kalpana Sharma and P. Rathi. Performance analysis of flooding and spin in wireless sensor networks. International Journal of Future Generation Communication and Networking, 7(3):25-36, 2014.

[13] H. Karl and A. Willig. Protocols and architectures for wireless sensor networks. John Wiley \& Sons, 2007.

[14] K.Karthikeyan and M.Kavitha. Comparative analysis of data centric routing protocols for wireless sensor networks. International Journal of scientific and Research Publications, 3, January 2013.

[15] A. Köpke, M. Swigulski, K. Wessel, D. Willkomm, P. K. Haneveld, T. Parker, O. Visser, H. S. Lichte, and S. Valentin. Simulating wireless and mobile networks in omnet++ - the mixim vision. In 1st International Workshop on OMNeT++ (hosted by SIMUTools 2008), Marseill, France, Mar. 2008.

[16] B. Latré, B. Braem, I. Moerman, C. Blondia, and P. Demeester. A survey on wireless body area networks. Wireless Networks, 17(1):1-18, 2011.

[17] B. Latré, B. Braem, I. Moerman, C. Blondia, E. Reusens, W. Joseph, and P. Demeester. A low-delay protocol for multihop wireless body area networks. In 4th Annual International Conference on Mobile and Ubiquitous Systems (MobiQuitous 2007), August 6-10, 2007, Philadelphia, $P A, U S A$, pages 1-8, 2007.

[18] W. Lou. An efficient n-to-1 multipath routing protocol in wireless sensor networks. In Mobile Adhoc and Sensor Systems Conference, 2005. IEEE International Conference on, pages 8 pp.-672, Nov 2005.

[19] S. Merugu, M. H. Ammar, and E. W. Zegura. Routing in space and time in networks with predictable mobility. Technical Report GIT-CC-04-07, Georgia Institute of Technology, 2004.

[20] S. Movassaghi, M. Abolhasan, and J. Lipman. A review of routing protocols in wireless body area networks. JNW, 8(3):559-575, 2013.

[21] S. Movassaghi, M. Abolhasan, J. Lipman, D. Smith, and A. Jamalipour. Wireless body area networks: A survey. IEEE Communications Surveys and Tutorials, 16(3):1658-1686, 2014.

[22] Ms.Venkateswari.R and D. Rani.S. Design of an energy efficient and delay tolerant routing protocol for wireless body area network. In International Journal on Computer Science and Engineering (IJCSE).

[23] J.-i. Naganawa, K. Wangchuk, M. Kim, T. Aoyagi, and J.-i. Takada. Simulation-based scenario-specific channel modeling for wban cooperative transmission schemes. IEEE Journal of Biomedical and Health Informatics, PP(99), May 2014.

[24] S.-Y. Ni, Y.-C. Tseng, Y.-S. Chen, and J.-P. Sheu. The broadcast storm problem in a mobile ad hoc network. In 5th annual ACM/IEEE international conference on Mobile computing and networking (MobiCom), Seattle, WA, USA, aug 1999.
[25] Z. Rehena, S. Roy, and N. Mukherjee. A modified spin for wireless sensor networks. In Communication Systems and Networks (COMSNETS), 2011 Third International Conference on, pages 1-4, Jan 2011.

[26] D. Singelée, B. Latré, B. Braem, M. Peeters, M. D. Soete, P. D. Cleyn, B. Preneel, I. Moerman, and C. Blondia. A secure low-delay protocol for wireless body area networks. Ad Hoc \& Sensor Wireless Networks, 9(1-2):53-72, 2010.

[27] A. Vahdat and D. Becker. Epidemic routing for partially connected ad hoc networks. Technical Report CS-2000-06, Department of Computer Science, Duke University, Apr. 2000.

[28] Z. Wang, E. Bulut, and B. Szymanski. Energy efficient collision aware multipath routing for wireless sensor networks. In Communications, 2009. ICC '09. IEEE International Conference on, pages 1-5, June 2009.

[29] W. Zeng, A. Arora, and N. B. Shroff. Maximizing energy efficiency for convergecast via joint duty cycle and route optimization. In INFOCOM 2010. 29th IEEE International Conference on Computer Communications, Joint Conference of the IEEE Computer and Communications Societies, 15-19 March 2010, San Diego, CA, USA, pages 16-20, 2010. 